\title{
Review of: "High-resolution structures of the actomyosin-V complex in three nucleotide states provide insights into the force generation mechanism"
}

\author{
Alf Månsson
}

Potential competing interests: The author(s) declared that no potential competing interests exist.

Review of paper: High-resolution structures of the actomyosin-V complex in three nucleotide states provide insights into the force generation mechanism published as preprint in BioRxiv by Pospich et al. ${ }^{1}$

Before starting the review, I wish to make clear to the reader that my perspective is that of a biophysicist (not a structural biologist) with particular emphasis on actin and myosin from striated muscle (e.g. ${ }^{2-5}$ ).

\section{Summary of key findings}

The paper by Pospich et al. ${ }^{1}$ is a comprehensive study that reports several high-resolution cryo-EM structures of myosin $\mathrm{V}$ in the strong-ADP and rigor states, together with a new post-rigor state. These reported structures have the potential to help elucidating the structural transitions in myosin that underlie the motor-cycle. One intriguing finding is that of conformational heterogeneities of biochemical states with either MgADP or no nucleotide at the active site. This result may suggest that conformational flexibility is of greater importance than previously thought for the structural changes along the motor cycle. However, in my mind there may be alternative interpretations (see below).

The data also give valuable insights into details of the actomyosin interface and highlight differences between myosin $\mathrm{V}$ and other myosins studied previously. Another interesting aspect of the paper is the highlighting of the role of the so called A-loop in the coordination of ADP binding by hydrophobic interactions and connecting events at the active site to other parts of the molecule.

Finally, the data demonstrate myosin-induced changes in the structure of F-actin. Of particular interest in the latter regard is the interactions between myosin-binding on the one hand and the actin-binding cyclic peptides phalloidin and jasplakinolide on the other. I believe that this issue should be discussed in greater detail in relation to a number of previous studies including results that have remained enigmatic for long time 6-14. 


\section{Overall assessment}

The paper is generally well and clearly written and the authors lay out the subject and the rationale of the study in an excellent way in the Introduction. The methods are well described and the study appears to be carefully performed. However, I think that the use of alpha-actin from skeletal muscle, in combination with a myosin motor that is expected to primarily interact with non-muscular actin isoforms, is an issue that requires some discussion. This is, not the least, important in view of key findings on the actin-myosin interface as well as allosteric changes in F-actin with potential functional implications.

The results are generally well described. However, for further clarity, the paper would benefit if some figures are somewhat amended and key terms are more precisely defined upon first use (see below). Most conclusions are fairly well supported by the data and discussed with generally appropriate citations of the published literature. However, there are some findings, as already mentioned above, where alternative interpretations would seem important to discuss. Furthermore, I think that the citations are somewhat too limited to the structural biology literature. In view of the implications of the results for the motor cycle as well as F-actin function, it seems appropriate with additional discussions of functional implications. More detailed comments and suggestions follow below.

\section{Clarity of terminology}

"Strong-ADP state"

The "strong-ADP state" should be more precisely defined in relation to existing actomyosin ADP states. Thus, is the "strong-ADP state" a state with strong ADP binding to myosin or strong myosin-binding to actin? This question is also relevant in relation to evidence (more or less explicit) for three ADP states in different myosins $(3,15-17)$. Thus, in models with Pi-release from the active site before force-generation, there would be one actomyosin-ADP state with the lever-arm in a pre-power-stroke conformation. This state would have ADP strongly bound but would bind actin rather weakly. Then there would be an ADP state after a first power-stroke $3,15,17,18$ presumably with intermediately strong ADP-binding and actinbinding). Finally there would be one actomyosin ADP-state after a second power-stroke. The latter has, for striated muscle myosin, been believed to have very weak ADP-binding with diffusion limited rates of ADP binding and dissociation ${ }^{19}$ and structural similarity to the rigor state (e.g. model in ${ }^{5}$ ).

\section{"Aged actomyosin"}

I presume that "aged actomyosin" refers to F-actin being aged as described. However, the concept would benefit from a precise and explicit definition to avoid any ambiguities.

\section{"Force sensitivity"}

Some transitions are referred to as "force sensitive". This concept should be precisely defined. E.g., would increased "force sensitivity" of a transition be equivalent to an increased change of the transition rate for a given change in force between each myosin molecule and the actin filament? 


\section{Modulation of F-actin structure by myosin binding}

It of interest to note that some of the findings reported on this issue in the paper of Pospich et al. ${ }^{1}$, may be of relevance to the understanding of long-term puzzling functional changes in F-actin upon myosin binding. Such changes have been found in several studies dating back to work by Yanagida et al. ${ }^{7}$ and earlier. A rather striking analogy to some of the present results appear in a study of Vikhorev et al. $^{2}$ with studies of both phalloidin-free and phalloidin-labelled F-actin. Thus Vikhorev et al. ${ }^{2}$ showed reduction in the persistence length of phalloidin-stabilized actin filaments propelled by myosin in the in vitro motility assay down to the same level as in the absence of phalloidin. In contrast, no effect of myosin-binding and filament-propulsion on the persistence length was seen with phalloidin free filaments. These results exhibit interesting similarities to the present findings with jasplakinolide-actin. Thus, it seems that binding of myosin to actin in the in vitro motility assay (even to a limited number of actin sites) abolished the effect of phalloidin on flexural rigidity while myosin binding had no effect on the flexural rigidity in the absence of phalloidin.

I am missing a discussion of the mentioned functional consequences of the toxin-binding in terms of effects on flexural rigidity. These consequences may find their explanation based on structural changes related to those reported here, despite somewhat different effects of phalloidin and jasplakinolide. In this context it also seems relevant to consider earlier studies e.g. ${ }^{8,9,14}$ relating flexural rigidity of actin filaments to Dloop conformation.

\section{Conformational heterogeneity of biochemical states in relation to force-generating mechanism}

On basis of the finding of conformational heterogeneity in a given biochemical state, the authors consider the possibility that mechanisms of the motor cycle may differ from the conventional view that assumes deterministic coupling between nucleotide state and structural state. The authors express this as follows: “The associated structural flexibility of myosin-V possibly initiates transitions between structural states by giving rise to short-lived in-between conformations with favorable nucleotide binding affinities. Interactions with a nucleotide would consequently not trigger the transition, but merely stabilize myosin in its transient conformation, thereby promoting the transition to a new structural ensemble state."

I wonder if the effect could have an appreciably simpler explanation based on ubiquitous thermal fluctuations of the protein.

This issue can be elucidated on the background of simple calculations like those below for myosin II of striated muscle (which I am more familiar with):

If one considers equipartition of energy for one degree of freedom, the following would apply: 
$k_{B} T=k_{C}<x>^{2}$

Here, $k_{B}$ is the Bolzmann constant, $T$ is the absolute temperature and $k_{C}$ is a stiffness of the myosin motor (in the direction along the actin filament) ${ }^{4}$. Now, with $\mathrm{k}_{\mathrm{B}} T \approx 410^{-21} \mathrm{~J}=4 \mathrm{pN} \mathrm{nm}$ and, assuming a stiffness of the cross-bridge lever arm of $2 \mathrm{pN} \mathrm{nm}^{-1}$, then

$<x>^{2}=2 m^{2}$.

Thus, an estimate of the standard deviation (SD) of the positional variability of the distal lever arm end in striated muscle myosin is $1.4 \mathrm{~nm}$. With a lever arm length of $9 \mathrm{~nm}$ in myosin II, this would correspond to 9 ${ }^{\circ}$ angular change or an $18^{\circ}$ difference between the lever arm positions corresponding to mean-1 SD and mean +1 SD. This angular spread (which is greater than reported in Fig, $9^{1}$ ) would be even greater for a myosin molecule with lower stiffness such as slow striated muscle myosin $\mathrm{II}^{18}$. I do not know what applies to myosin $\mathrm{V}$ in this regard but it seems unlikely that the angular stiffness should be appreciably higher than for myosin II.

Therefore, in view of the above calculations the observed heterogeneity is broadly consistent with thermal fluctuations. A difference is of course that the present study seems to imply that the different conformational states are metastable/stable so that they can be captured by cryo-EM with the current preparation and imaging procedure. However, I wonder if not thermal fluctuations alone, without any metastable intermediate states, would be sufficient to account for the heterogeneity. I presume, for instance, that this would be the case if each conformational sub-state, in reality, represents an average of a continuous spectrum of neigbouring conformations populated by thermal fluctuations. As far as I understand such continuous conformational variability must exist when freezing is applied and should somehow appear in the results? It would be of interest to know what the authors think about this idea. Another possibility, to really produce metastable states of conformations populated by thermal fluctuations would be if some myosin structural states are captured e.g. by surface interactions. From careful reading of the Methods, such complications do not seem unlikely. First, F-actin is claimed to be saturated with myosin (line 809). This suggests that a large number of the myosin molecules should be close to the surface because it is stated on lines 781-782 that myosin was added after actin filament deposition on the surface.

\section{Minor issues}

Fig. 1. The B panel is unclear: it is hard to distinguish the residue labeling as well as the switch labeling (particularly Switch II). 
Fig. 3. Similar problem as in Fig. 1. The B panel is unclear: it is hard to distinguish the residue labeling.

Lines 618-619: "bound to a second binding site consisting of S153, T197, S203 and E461" This is, as far as I can see, not consistent with what is stated by Llinas et $\mathrm{al}^{20}$ for this second binding site.

Line 886: "no 3D mask was used"

\section{References}

1. Pospich, S., Sweeney, H.L., Houdusse, A. \& Raunser, S. High-resolution structures of the actomyosin-V complex in three nucleotide states provide insights into the force generation mechanism. BioRxiv (2021).

2. Vikhorev, P.G., Vikhoreva, N.N. \& Mansson, A. Bending flexibility of actin filaments during motorinduced sliding. Biophys. J. 95, 5809-5819 (2008).

3. Albet-Torres, N. et al. Drug effect unveils inter-head cooperativity and strain-dependent ADP release in fast skeletal actomyosin. J. Biol. Chem. 284, 22926-22937 (2009).

4. Månsson, A., Usaj, M., Moretto, L. \& Rassier, D.E. Do Actomyosin Single-Molecule Mechanics Data Predict Mechanics of Contracting Muscle? International journal of molecular sciences 19 (2018).

5. Rahman, M.A., Usaj, M., Rassier, D.E. \& Månsson, A. Blebbistatin Effects Expose Hidden Secrets in the Force-Generating Cycle of Actin and Myosin. Biophys. J. 115, 386-397 (2018).

6. Yanagida, T. \& Oosawa, F. Effect of myosin on conformational changes of F-actin in thin filament in vivo induced by calcium ions. Eur. J. Biochem. 56, 547-556 (1975).

7. Yanagida, T., Nakase, M., Nishiyama, K. \& Oosawa, F. Direct observation of motion of single F-actin filaments in the presence of myosin. Nature 307, 58-60 (1984).

8. Orlova, A. \& Egelman, E.H. A conformational change in the actin subunit can change the flexibility of the actin filament. J. Mol. Biol. 232, 334-341 (1993).

9. Orlova, A. \& Egelman, E.H. Structural dynamics of F-actin: I. Changes in the C terminus. J. Mol. Biol. 245, 582-597 (1995).

10. Orlova, A., Prochniewicz, E. \& Egelman, E.H. Structural dynamics of F-actin: II. Cooperativity in structural transitions. J. Mol. Biol. 245, 598-607 (1995).

11. Galkin, V.E., Orlova, A., Schroder, G.F. \& Egelman, E.H. Structural polymorphism in F-actin. Nature structural \& molecular biology 17, 1318-1323 (2010).

12. Galkin, V.E., Orlova, A. \& Egelman, E.H. Actin filaments as tension sensors. Current biology : CB 22, R96-101 (2012).

13. Kozuka, J., Yokota, H., Arai, Y., Ishii, Y. \& Yanagida, T. Dynamic polymorphism of single actin molecules in the actin filament. Nature Chemical Biology2, 83-86 (2006).

14. Chu, J.W. \& Voth, G.A. Coarse-grained modeling of the actin filament derived from atomistic-scale simulations. Biophys. J. 90, 1572-1582 (2006). 
15. Veigel, C., Wang, F., Bartoo, M.L., Sellers, J.R. \& Molloy, J.E. The gated gait of the processive molecular motor, myosin V. Nature cell biology 4, 59-65 (2002).

16. Mentes, A. et al. High-resolution cryo-EM structures of actin-bound myosin states reveal the mechanism of myosin force sensing. Proc. Natl. Acad. Sci. U. S. A.115, 1292-1297 (2018).

17. Hwang, Y., Washio, T., Hisada, T., Higuchi, H. \& Kaya, M. A reverse stroke characterizes the force generation of cardiac myofilaments, leading to an understanding of heart function. Proc. Natl. Acad. Sci. U. S. A. 118 (2021).

18. Capitanio, M. et al. Two independent mechanical events in the interaction cycle of skeletal muscle myosin with actin. Proc. Natl. Acad. Sci. U. S. A.103, 87-92 (2006).

19. Nyitrai, M. et al. What limits the velocity of fast-skeletal muscle contraction in mammals? J. Mol. Biol. 355, 432-442 (2006).

20. Llinas et al. How actin initiates the motor activity of myosin. Dev Cell. 33, 401-412 (2015) 